\title{
Vous avez dit : « Mettre en œuvre le principe de laïcité de manière éthique et responsable »?
}

\section{Gilles Moutot et Jean-Pierre Véran}

\section{(2) OpenEdition \\ 1 Journals}

\section{Édition électronique}

URL : http://journals.openedition.org/trema/2699

DOI : 10.4000/trema.2699

ISSN : 2107-0997

\section{Éditeur}

Faculté d'Éducation de l'université de Montpellier

\section{Édition imprimée}

Date de publication : 1 avril 2012

Pagination : $22-41$

ISSN : 1167-315X

\section{Référence électronique}

Gilles Moutot et Jean-Pierre Véran, « Vous avez dit : « Mettre en œuvre le principe de laïcité de manière éthique et responsable » ? », Tréma [En ligne], 37 | 2012, mis en ligne le 01 avril 2014, consulté le 30 avril 2019. URL : http://journals.openedition.org/trema/2699 ; DOI : 10.4000/trema.2699

Ce document a été généré automatiquement le 30 avril 2019

Trema 


\section{Vous avez dit : « Mettre en œuvre le principe de laïcité de manière éthique et responsable »?}

Gilles Moutot et Jean-Pierre Véran

Il est plus facile d'élever un temple que d'y faire descendre l'objet du culte.

S. BECKETT, L'Innommable. 
Dans son discours prononcé au Puy-enVelay le 3 mars 2011, le Président de la République française déclare: «La République, mes chers compatriotes, est laïque. Elle appartient donc à tous les citoyens sans distinction aucune [...]».

Ce propos paraît s'inscrire dans une continuité constitutionnelle.

L'article 1 de l'actuelle Constitution de la

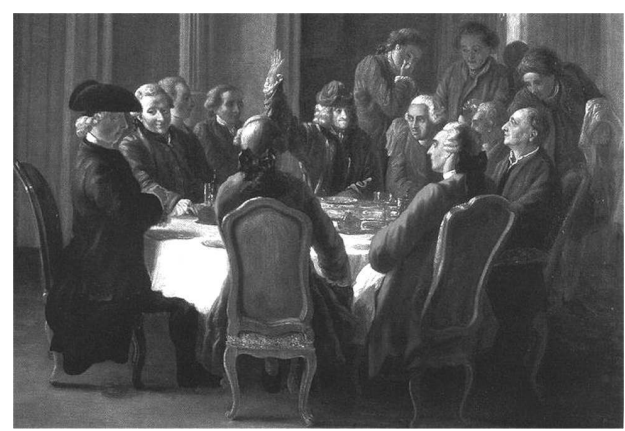
Ve République indique que «la France est une République indivisible, laïque, démocratique et sociale. Elle assure l'égalité devant la loi de tous les citoyens sans distinction d'origine, de race ou de religion. Elle respecte toutes les croyances ».

Le préambule de la Constitution de 1946 rappelle que « la Nation garantit l'égal accès de l'enfant et de l'adulte à l'instruction, à la formation professionnelle et à la culture. L'organisation de l'enseignement public gratuit et laïque à tous les degrés est un devoir de l'État ».

CONDORCET, dans son Rapport et projet de décret sur l'organisation générale de l'instruction publique, écrit en 1792 :

La Constitution, en reconnaissant le droit qu'a chaque individu de choisir son culte, en établissant une entière égalité entre tous les habitants de la France, ne permet point d'admettre, dans l'instruction publique, un enseignement, qui, en repoussant les enfants d'une partie des citoyens, détruirait l'égalité des avantages sociaux et donnerait à des dogmes particuliers un avantage contraire à la liberté des opinions (CONDORCET, 1792).

3 Cette continuité apparente ne fait illusion pour personne. La laïcité est en effet devenue un enjeu, voire un otage du débat politique, et la diversité de ses acceptions chez les responsables politiques comme parmi les penseurs médiatiques qui animent le débat public brouille singulièrement la tâche du personnel de l'éducation nationale. L'article L $11.1 \mathrm{du}$ code de l'éducation indique en effet qu'»outre la transmission des connaissances, la Nation fixe comme mission première à l'école de faire partager aux élèves les valeurs de la République ». Et il précise : « Dans l'exercice de leurs fonctions, les personnels mettent en œuvre ces valeurs ».

4 Comment mettre en œuvre aujourd'hui dans l'exercice quotidien de son métier d'enseignant, de conseiller principal d'éducation, de personnel de direction, la laïcité quand elle prend, dans le débat public comme dans les propos du président de la République, des acceptions paradoxales? Nous aborderons d'abord quelques questions que peuvent se poser les professionnels de l'Éducation nationale dans ce contexte avant de tenter de poser un diagnostic sur cet étrange moment politique.

\section{Sacralisation de l'École et religion de la laïcité ?}

5 L'auteur de l'Éloge des frontières, pour qui « là où il y du sacré il y a une enceinte » (DEBRAY, 2010, p. 36) s'est fait, dans Le Moment fraternité notamment, l'éloquent avocat de la sacralisation des savoirs et de l'École : «Remise à niveau, l'école a perdu son statut de "lieu à part", où s'apprend, à l'écart des familles, la citoyenneté républicaine » (Debray, 2009, p. 52). 
6 Il n'est pas vain de constater que des philosophes, comme Henri PENA-RUIZ, estiment, dans cet esprit, qu'on ne peut qualifier la laïcité, parler de laïcité ouverte ou plurielle revenant à nier la laïcité :

Une telle laïcité n'est ni dure ni molle : elle correspond à l'affranchissement de la sphère publique par rapport à toute tutelle religieuse; entre un tel affranchissement et une réattribution d'emprises publiques à la religion, il n'y a pas de troisième voie. L'alternative est donc bien : affirmation ou négation de la laïcité ( PENA-RUIZ, 2001, p. 79).

7 Cette volonté de sacralisation est sans doute une clef de lecture utile de la loi no 2004-228 du 15 mars 2004 encadrant, en application du principe de laïcité, le port de signes ou de tenues manifestant une appartenance religieuse dans les écoles, collèges et lycées publics.

8 L'article 1 de cette loi stipule :

Il est inséré, dans le code de l'éducation, après l'article L. 141-5, un article L. 141-5-1 ainsi rédigé : «Art. L. 141-5-1. - Dans les écoles, les collèges et les lycées publics, le port de signes ou tenues par lesquels les élèves manifestent ostensiblement une appartenance religieuse est interdit. Le règlement intérieur rappelle que la mise en œuvre d'une procédure disciplinaire est précédée d'un dialogue avec l'élève ».

9 Alors que le principe de stricte neutralité religieuse, politique, commerciale s'applique aux agents du service public, afin de respecter "une entière égalité entre tous les habitants de la France », cette loi applique cette neutralité aux élèves également. Une seule fois auparavant, sous le gouvernement de Léon BLUM formé en 1936, une circulaire du ministre Jean ZAY, dans le contexte d'affrontements violents entre lycéens appartenant aux ligues factieuses et aux partis du front populaire, avait recommandé une stricte neutralité politique aux élèves. Il est frappant de constater, d'autre part, que la loi du 15 mars 2004 n'a retenu qu'une seule des propositions de la commission STASI chargée par le président de la République de contribuer à son élaboration. On se souvient que le rapport remis au président de la République le 11 décembre 2003 en comportait bien d'autres, comme, par exemple, «faire des fêtes religieuses de Kippour et de l'Aïd el Kebir des jours fériés dans toutes les écoles de la République ». Ce choix exclusif d'interdire aux élèves la manifestation ostensible d'une appartenance religieuse, interdiction qui vise implicitement le port d'un voile par des élèves de confession musulmane, d'un turban par des élèves sikhs ou d'une kippa par des élèves de confession juive, ne renforce-t-il pas la lecture de cette loi comme procédant d'une interprétation religieuse de la laïcité ?

10 En mars 2011, le ministre de l'Éducation nationale a formulé le souhait de voir ce principe appliqué également aux parents encadrant des sorties scolaires :

Il me semble nécessaire de rappeler que les parents qui accompagnent des élèves, inscrits dans une école ou un établissement scolaire publics, au cours d'activités ou de sorties scolaires, participent ce faisant à l'action éducative et plus généralement au service public de l'éducation. En faisant acte de candidature, ils sont amenés à participer directement au service public de l'éducation en se plaçant dans une situation comparable à celle des agents publics vis-à-vis des enfants qu'ils encadrent et acceptent donc de se soumettre aux principes fondamentaux de ce service public.

11 On peut légitimement s'interroger, en tant qu'enseignant, personnel d'éducation ou de direction, sur le sens d'une telle mise en œuvre du principe de laïcité. À écouter certains raisonner par analogie - «quand on rentre dans une mosquée, on doit enlever ses chaussures. De même, quand une élève rentre dans une classe, elle doit enlever son foulard»-, il semble bien que l'on assiste à une paradoxale sacralisation de la laïcité. 
Cette sacralisation est-elle compatible avec l'ambition du service public d'éducation d'être hospitalier à tous les enfants et ne risque-t-elle pas, comme le redoutait CONDORCET, de « repousser les enfants d'une partie des citoyens » (CONDORCET, 1792) ? Ne pourrait-on pas s'interroger sur la nécessité de laïciser la laïcité?

\section{Identité nationale et christianisation de la laïcité ?}

12 Le thème de l'identité nationale a traversé l'actuel quinquennat du président de la République. Le discours que ce dernier a prononcé au Puy-en-Velay en mars 2011 éclaire nettement son point de vue. Saluant « un formidable viaduc spirituel lancé vers le Ciel », il affirme :

Chartres, Amiens, Reims, Strasbourg, Paris, aucune de ces villes ne serait aujourd'hui ce qu'elle est aux yeux des Français et aux yeux du reste du monde sans ces cathédrales vers lesquelles convergent toujours fidèles et touristes [...]. En disant cela, je ne fais que rappeler une évidence : l'apport de la chrétienté à notre civilisation [...]. La chrétienté nous a laissé un magnifique héritage de civilisation et de culture [...]. Je ne fais pas de prosélytisme, je regarde simplement l'Histoire de notre pays [...]. Ce baptistère serait donc l'exact contemporain du sacre de Clovis et donc de la naissance de la France. Ce n'est pas rien! [...] Construire l'Europe de demain, c'est au fond continuer à suivre le chemin tracé il y a plus de mille ans par les premiers pèlerins de Saint-Jacques de Compostelle. N'étaient-ils pas les premiers européens, ces pèlerins-là ? [...] Ils ne parlaient pas la même langue mais il partageaient la même foi ou en tout cas la même espérance [...].

Et il conclut :

J'étais venu vous dire que la France a une identité dont elle peut être fière, mais ici, au Puy-en-Velay, peut être plus qu'ailleurs, il est évident que la France a aussi une âme.

Ces propos trouvent leur préfiguration dans le rapport remis par le vice-président de l'Assemblée nationale, François BAROIN, en mai 2003, au Premier ministre Pour une nouvelle laïcité. L'auteur y promeut une laïcité "culturelle et identitaire», en observant que la laïcité est "questionnée et contestée", écrit-il, "en particulier dans le monde musulman » et par «certaines populations immigrées, qui, issues d'une culture non laïque et non démocratique, ne perçoivent pas le sens de ce principe ».

Comme le remarque Jean BAUBÉROT dans une tribune parue dans Libération le 29 mars 2011, « depuis 2007, c'est le haut Conseil à l'intégration (HCI) ${ }^{1}$ qui fait des propositions sur la laïcité. Cette dernière se réduit à être un passeport que de "nouveaux Français" devraient acquérir pour revêtir l'identité française. Les questions qui concernent l'ensemble des Français sont exfiltrées de la laïcité » (вачвÉRoт, 2011). Il note également que, dans le discours du Puy-en-Velay, le Président affirme qu'«il est dangereux d'amputer sa mémoire", alors que le passé national qu'il invoque fait silence sur les croisades, les guerres de religions, la révocation de l'Édit de Nantes, l'esclavage, la sujétion des musulmans dans l'empire colonial. L'identité nationale promue dans le discours du Puy serait donc pour Jean BAUBÉROT une «identité patrimoniale non conflictuelle imaginaire » (ВАUBÉROT, 2011).

15 Le lien établi entre laïcité et identité nationale fortement marquée par l'apport de la chrétienté («l'âme de la France») se traduit par la persévérance à légiférer ou réglementer au nom de la laïcité en visant l'éradication de l'école, des services publics, voire de l'espace public de signes d'appartenance religieuse - et en se préoccupant 
principalement d'une religion. Cela ne constitue-t-il pas une sorte de renversement du principe de laïcité, principe politique établi pour bannir toute discrimination pour raison religieuse notamment?

Cette laïcité de contrainte exercée à l'égard d'une partie de la population n'aboutirait-elle pas à renforcer des réactions que nous observons chez certains de nos élèves français par leur état civil: graffiti célébrant un autre pays, port de $t$-shirts affirmant une appartenance nationale autre, opposition dans le discours destiné aux adultes du "vous » (les Français, à l'identité nationale bien marquée par un refus d'hospitalité à certains) à un « nous » identitaire de relégation et de protestation?

Ces questions se posent d'autant plus que la vigilance d'associations familiales chrétiennes à l'égard des programmes d'enseignement ne semble pas susciter la même réaction de sourcilleuse laïcité, comme on vient de le voir à l'occasion de la parution du nouveau programme de SVT qui aborde en classe de première la théorie des genres ${ }^{2}$. Elles dénoncent "l'idéologie du genre au lycée » comme « une attaque en règle contre la loi morale naturelle, rejetant toute signification anthropologique et éthique à la différence naturelle des sexes ${ }^{3} »$.

Dans ces conditions, le moins que l'on puisse dire c'est que chaque enseignant, conseiller principal d'éducation, personnel de direction a légitimement de quoi s'interroger sur la manière d'agir conformément au principe de laïcité dans l'exercice quotidien de ses missions, pendant lequel il est censé «mettre en œuvre les valeurs de la République » en ayant, bien entendu, à cœur d'« agir en fonctionnaire de l'État et de manière éthique et responsable ${ }^{4}$. Cette question est d'autant plus épineuse qu'elle est pour ainsi dire à double fond, comme si les confusions entourant aujourd'hui le principe de laïcité (confusions dont tout porte à croire qu'elles sont, ici ou là, très sciemment entretenues, et forment ainsi en elles-mêmes un sujet d'inquiétude politique) mettaient, du même coup, violemment à nu l'indigence du "cadre » institutionnel censé en guider l'inscription concrète dans les métiers de l'éducation et de la formation. C'est cette circularité délétère qu'on voudrait analyser maintenant.

\section{Tartuffe technocrate}

«Confusion", disions-nous : si le mot nous semble approprié, c'est dans la mesure où il touche autant à la manière qu'à la matière. Les exemples sur lesquels les pages précédentes attirent l'attention ne laissent aucun doute là-dessus : c'est au mépris de la laïcité qu'on prétend en célébrer les principes - tel celui de la neutralité de l'État vis-à-vis des cultes (de tous les cultes).

Qu'on en juge : l'«affaire » des manuels de SVT de la classe de première poussant leur conformité avec les programmes officiels jusqu'à l'audace de mentionner la dimension culturellement construite de l'identité sexuelle ne s'est pas arrêtée à la lettre ouverte de Mme Boutin, sommant le ministre de l'Éducation nationale de sauver les adolescents du pays d'une "prise d'otages" (selon la rhétorique dont raffolent ceux qui n'ont vraisemblablement jamais été l'otage de qui que ce soit) par des "groupes minoritaires " (que, pour le coup, on se garde de nommer). De fait, si, selon des déclarations rapportées par l'AFP le 31 août 2011, Luc CHATel s'est refusé à demander le retrait des manuels incriminés par quelque quatre-vingt députés UMP, ceux-ci auront eu au moins la satisfaction de voir Jean-François CoPÉ soutenir publiquement leur cause. Le secrétaire 
général de l'UMP croit en effet savoir que la théorie du genre, qui selon lui « est discutée par des personnes mais combattue par d'autres ", n'est pas une "vérité scientifique » et qu'à ce titre elle ne devrait pas être "enseignée ». Disons-le: on ne gaspillera pas du temps à demander quelle idée de la science on se fait ici pour que l'interlocution entre sciences humaines, sciences sociales et sciences de la vie soit inconcevable, et pour que débats et controverses semblent, contre la tradition même de la pensée scientifique, définir le contraire de la science. De toute façon, l'enjeu est ailleurs : le recours indigent à un prétendu arbitrage scientifique masque mal le souci d'adresser, pour user d'un jargon aussi dominant que piteux, un « signal fort » à une «frange de l'électorat », représentée par le courant dit de la « Droite populaire ». La manœuvre n'est guère nouvelle mais, en l'occurrence, elle se paye, intellectuellement et moralement, au prix fort: le chef de file du parti présidentiel (et, dans une moindre mesure, le ministre de l'Éducation nationale, qui, s'il a renoncé à interdire, a toutefois tenu à dire sa "réserve ", en des termes proches de ceux de M. copé) prend fait et cause, sous couvert de science, pour une définition prétendument « naturelle » de l'identité sexuelle et, par là même, pour une soumission de l'information biologique à une représentation de l'homme et de la société dont les coordonnées religieuses n'échappent à personne.

Bref, comme le résume Éric FASSIN, « l'aile la plus réactionnaire se fait bien entendre non seulement dans les débats sur la nationalité, mais aussi en matière de sexe » (FASSIN, 2011). En sorte que l'ardeur avec laquelle ces voix se défendent de cautionner quelque conviction homophobe est à peu près aussi convaincante que le pathos avec lequel le président de la République se disait hier, à Latran, le défenseur de «l'attachement des Français à la laïcité », et aujourd'hui, au Puy-en-Velay, le garant d'une République qui, « mes chers compatriotes [...] appartient [...] à tous les citoyens sans distinction aucune ».

De l'un à l'autre de ces discours, se confirme l'escroquerie intellectuelle baptisée «laïcité positive » - et l'on gagne à se rappeler la réaction de Jean-Claude MONOD aux glissements opérés par le discours présidentiel, publiée dans Le Monde en janvier 2008. Il y rappelait les traits fondamentaux de l'État laïque, défini par Ferdinand BuIsson, dans l'article «Laïcité » du Dictionnaire de pédagogie et d'instruction primaire, comme « neutre entre tous les cultes, indépendant de tous les clergés, dégagé de toute conception théologique » (F. BUISSON, cité par J.-C. MONOD, 2008). À partir de cette référence, l'auteur soulignait la duplicité d'une prétendue "défense " de la laïcité consistant essentiellement à placer, dans le domaine de la transmission des valeurs morales, l'instituteur républicain audessous du prêtre et à célébrer «la vertu théologale d'espérance en son acception religieuse, à laquelle il [Nicolas SARKOZY] confère une plus grande valeur qu'aux espérances séculières » (MONOD, 2008). Si cela caractérise une laïcité désormais affublée de l'épithète "positive ", autant dire que la consistance de celle-ci est, pour emprunter une expression d'ADORNO, celle d'un "concept mollusque» - et sa fonction purement stratégique : on laisse croire que la conception de la laïcité historiquement repérée par la loi de 1905 serait, vis-à-vis des religions, «négative» (entendons : hostile, répressive), alors que celle-ci, justement, est neutre et presque "amicale» au sens où l'entendait BUISSON. Posant le principe de l'égale liberté de conscience, elle garantit à toutes les religions le libre exercice du culte en même temps qu'elle défend le droit de chacun (athée, agnostique, croyant) de ne subir nul prosélytisme, que celui-ci émane de l'État ou des Églises (MONOD, 2008). Une fois l'art de la distorsion rétrospective poussé jusqu'à la falsification historique, ne devrait-on pas au moins cesser d'être hypocrite? Telle était, 
en janvier 2008, la conclusion à laquelle parvenait Jean-Claude MONOD et qu'on ne peut relire aujourd'hui sans en saluer, hélas, la dimension prophétique :

Plutôt que de prétendre réaliser une légère inflexion par rapport à la laïcité républicaine de 1905, à laquelle, entre deux piques, on rend un hommage bien formel tout en l'amputant d'un principe fondamental, le président et ses conseillers en la matière devraient dire franchement qu'ils abandonnent le principe républicain de la neutralité de l'État et de ses représentants, dans la sphère publique, en matière confessionnelle (MONOD, 2008).

À la lumière de ces lignes, la trajectoire qui mène de Latran au Puy-en-Velay prendrait presque des allures de success story: l'histoire d'un président qui s'est appliqué à maintenir, et à renforcer, le cap d'une expression « décomplexée » de ses convictions (làdessus, il est vrai, la couleur fut maintes fois annoncée - La Princesse de Clèves en fit même deux fois les frais, avant et après l'élection de son contempteur). Là où celles-ci touchent à sa foi religieuse (catholique, en l'occurrence), elles lui paraissaient déjà, en 2007, assez indiscutables pour mériter un régime de faveur: saluant alors la récente loi de 2004 interdisant aux élèves le port de signes religieux ostensibles à l'école publique (dite, par un raccourci éloquent, « loi sur le voile»), Nicolas SARKOZY ne ressentait, pour autant, nul embarras à "manifester ostensiblement sa foi catholique dans un discours public », et ainsi à suggérer qu'une telle manifestation «n'est pas incompatible avec la laïcité lorsqu'il s'agit du catholicisme (du président, du moins), tandis qu'elle est inacceptable pour l'islam (des lycéennes) ${ }^{5}$ " (MONOD, 2008). En 2011, un débat sur la nationalité plus tard, on est indubitablement passé à la vitesse supérieure : la foi du Président ne se contente plus de signaler l'excellence supposée d'un individu, elle exprime "l'âme » même du pays, d'une France à laquelle participent, plus authentiquement que les autres citoyens, et " au Puy-en-Velay plus qu'ailleurs », ceux qui inscrivent leurs pas dans ceux des "pèlerins de Saint-Jacques de Compostelle ». Voilà de quoi liquider le principe de la liberté de conscience, condition de possibilité de l'espérance très séculière en l'établissement d'un monde commun: celui qu'ont en partage, comme le rappelle l'étymologie du terme " laïcité », les membres du laos - en grec, le peuple considéré dans sa plus grande extension, formant le but du gouvernement démocratique («pour le peuple ») quand bien même les acteurs du gouvernement ("par le peuple») forment le groupe restreint du demos. Sans doute l'histoire des formes de cette restriction est-elle celle d'une « privatisation » de la sphère publique par des groupes ou castes déterminés en sorte que la démocratie menace sans cesse de n'exister que sous la forme d'« États de droit oligarchiques », pour reprendre une formule de Jacques RANCIÈRE (2005, p. 81 sq.). C'est justement pourquoi la laïcité, pour autant que s'y attache la question d'une réconciliation du laos et du demos, comporte une affinité élective avec l'utopie : l'espoir dans la possibilité d'un "universel» qui, affranchi tant de "l'indifférence aux différences " que de l'hypostase d'une différence (et donc d'une identité) particulière, s'accomplirait dans la « conjonction des différences » (ADORNO, 1970, posth., p. 263). C'est bien d'un tel espoir que, en effaçant la complexité du rapport de la France à son héritage chrétien pour trouver dans celui-ci l'instrument d'une définition essentialiste du «Français », on opère brutalement la négation - dans un tel contexte, l'expression « identité nationale » ne dit rien d'autre. Comment s'étonner, dès lors, qu'aujourd'hui une représentante patentée de l'extrême droite ne manque nulle occasion de poser en Jeanne d'ARc de la laïcité?

Sans doute n'est-ce pas là la moindre des raisons pour lesquelles le libellé de la première des «dix compétences à acquérir par les professeurs, documentalistes et conseillers 
principaux d'éducation pour l'exercice de leur métier " puisse paraître, aux yeux des concernés, au mieux futile, au pire cynique. Avant même la distribution, en « connaissances », « capacités » et «attitudes », de "contenus » dont la pleine maîtrise semble définir, au-delà du seul alignement européen des "référentiels ", quelque chose comme le nouvel idéal du moi de l'enseignant du XXIe siècle, le texte introductif, égrenant une litanie de qualités qui, ainsi aplaties par les constructions au présent de l'indicatif, n'en sont même plus, offre d'emblée quelques occasions de rire jaune. Ainsi : « Il [le professeur / le documentaliste / conseiller principal d'éducation] connaît et fait respecter les principes de la laïcité, notamment la neutralité ». Le lecteur a encore suffisamment en tête le parcours restitué ci-dessus - de Latran au Puy-en-Velay, en passant par les bureaux respectifs du secrétaire général de l'UMP et du ministre de l'Éducation nationale - pour que la citation de cette phrase se passe de longs commentaires. L'enseignant-modèle de l'arrêté de 2010 est soumis au même régime que les jeunes filles musulmanes dans le discours de 2007 du président de la République : le régime du « cela vaut pour vous, pas pour moi » - voilà tout.

En revanche, qu'on nous permette de mobiliser ici le souvenir d'une expérience de formation, qui nous semble de nature à prolonger l'analyse. L'anecdote - qui pourrait donc être un peu plus que cela - se situe dans le cadre suivant: les cours rendus nécessaires par l'introduction, dans les concours de recrutement des enseignants, documentalistes et $\mathrm{CPE}$, d'une épreuve relative, lors des oraux d'admission, à cette fameuse " première compétence ${ }^{6}$ " (la " compétence éthique ", selon la contraction qui s'impose couramment). S'efforçant d'en produire autant que possible une intelligence critique, au moyen notamment d'une mise en perspective philosophique et historique, on a pu, entre autres matériaux, proposer aux étudiants des extraits significatifs de la circulaire adressée aux instituteurs, le 17 novembre 1883, par Jules FERRY, alors ministre de l'Instruction publique - circulaire rendue fameuse sous la désignation de « Lettre aux instituteurs». Sans doute l'importance de ce texte tient-elle aux tensions qui le traversent : celles-là mêmes auxquelles les instituteurs se trouvent confrontés depuis que la loi du 28 mars 1882 leur a enjoint de dispenser un « enseignement moral et civique » en évitant cependant de tomber, ce faisant, dans «l'enseignement de tout dogme particulier ». Comment faire ? Comment, autrement dit, concevoir et, surtout, mettre en pratique une instruction morale laïque - séparée de l'instruction religieuse et ayant sa valeur propre? Tel est le point sur lequel Jules FERRY vient s'adresser non pas même, comme le suggère la formule passée à la postérité, « aux instituteurs ", mais bien à chaque instituteur (« Monsieur l'Instituteur [...] ») afin de dire pourquoi, selon lui, « la tâche n'est ni au-dessus de vos forces ni au-dessous de votre estime ${ }^{7} »$. Or, des propositions avancées à cette fin par Jules FERRY, celles formant le passage peut-être le plus célèbre de la lettre suscitent très souvent un fort intérêt de la part des étudiants. Ces lignes proposent, selon les termes de Jules FERRY, une « règle pratique ", et il faut prendre l'expression à la lettre : ni simple rappel d'un principe, ni invitation aux accommodements empiriques, mais bien la recherche d'une articulation entre, pour le dire vite, la théorie et la pratique, la nécessaire référence à une normativité (et, au-delà, à un ordre de valeurs) et la prise en compte, non moins nécessaire, des conditions, inévitablement changeantes et singulières, de l'action. Le résultat prend la forme d'une maxime prudentielle, invitant l'enseignant à choisir les propos qu'il s'apprête à tenir devant ses élèves en fonction du souci de ne pas heurter les convictions de leurs familles. Lisant aujourd'hui, donc, ces recommandationslà, quelle est la réaction, fréquemment enregistrée, des étudiants ? Elle consiste à dire, en 
substance, qu'un tel propos est beaucoup plus proche d'eux, bien plus audible, bien plus pertinent que la somme d'énumérations proposées dans l'en-tête, puis dans les listes de connaissances, capacités, attitudes composant aujourd'hui l'énoncé de la première « compétence » des professeurs, documentalistes et conseillers principaux d'éducation.

Qu'on nous comprenne bien: il n'est nullement question, ici, de faire entendre la rengaine du "c'était mieux avant " - expression d'un « néo-républicanisme » douteux, toujours prêt à fournir la rhétorique moraliste ${ }^{8}$ dont raffolent ceux qui, dans le même temps, ne dédaignent pas d'aligner l'école des «compétences» sur le modèle d'une compétition brutale entre les individus - vae victis. Il ne s'agit pas non plus de disqualifier, unilatéralement, les contenus rangés aujourd'hui sous la rubrique « Agir en fonctionnaire de l'État et de manière éthique et responsable ». Il n'y a, après tout, rien de condamnable à rappeler qu'un professeur doit connaître, par exemple « les valeurs de la République et les textes qui les fondent " - mais cela ne désigne-t-il pas une exigence s'imposant en fin de compte à tout citoyen (même, on est gêné de se sentir obligé de le rappeler, au vu d'événements évoqués ci-dessus, à celui d'entre eux qui se trouve en charge des plus hautes fonctions de l'État)? Inversement, on n'aura pas l'ingénuité d'ignorer les profondes ambivalences qui traversent le texte de Jules FERRY, et qui pour une part communiquent avec les aspects idéologiques les plus douteux de la IIIe République: certes, FERRY assure aux destinataires de sa lettre qu'ils ne sont " point les apôtres d'un nouvel évangile » - «mais quand même », ne peut s'empêcher de murmurer le lecteur d'aujourd'hui, tant l'usage de la prétérition crible le texte d'emprunts au lexique religieux (" théologien », " mission », " apostolat », etc.) ; surtout, la relative facilité avec laquelle, selon l'auteur, l'enseignant saura, en fin de compte, puiser dans les ressources d'une morale consensuelle, "la bonne et antique morale de nos pères", expression, tout compte fait, de la "sagesse du genre humain ", recueil de "ces idées d'ordre universel ", trahissent évidemment une sorte de foi dans quelque chose comme un universel facile, peu enclin à réfléchir sur la particularité de sa genèse historique, et assez ignorant de ses taches aveugles pour justifier, dans l'esprit du même Jules FERRY, l'exportation coloniale de la « civilisation».

Il reste pourtant que, encore négativement obnubilé par la figure cléricale du maitre, l'auteur de la circulaire de novembre 1883 n'y cherche pas moins à dégager les traits fondamentaux, et la légitimité propre, de l'enseignant dans une école publique laïque. La neutralité de celui-ci procèdera donc justement, et d'abord, d'une neutralisation de la recherche des positions hiérarchiques au sein d'un improbable palmarès spirituel - celuilà même que, quelque cent vingt-cinq ans plus tard, un croyant " décomplexé » devenu président de la République se pique de remettre au goût du jour. Il reste, également, que, si idéalisée soit-elle, la référence à une morale "universelle » a guidé, et contribué à rendre effective (certes non sans conflits, défiances ou anathèmes lancés de part et d'autre ${ }^{9}$ ) cette prudente neutralité laïque qu'attendaient de l'École des consciences athées, agnostiques ou même croyantes - mais, pour autant, pas moins méfiantes envers le prosélytisme de l'Église ${ }^{10}$.

Par là s'entrevoit l'une des principales raisons de l'intérêt que les étudiants portent à cette lettre : elle tient à la liaison étroite de la forme et du fond. Entendons : précisément parce que (non sans ambivalences, non sans de lourds présupposés idéologiques, au nombre desquels figurent en bonne place, outre ceux déjà mentionnés, les rapports de genre) le principe de neutralité de l'enseignant est ici dégagé en réponse aux inquiétudes nourries par " Monsieur l'instituteur ", il est, et doit être, adressé à un sujet. Voilà ce que 
peuvent se dire, aujourd'hui, ceux qui, personnels d'éducation ou étudiants, et comme tels visés par le référentiel des "dix compétences ", ont l'occasion de placer celui-ci en face de la lettre de Jules FERRY du 17 novembre 1883. Pour peu qu'ils aient l'occasion d'y faire halte quelques instants et d'en appréhender le contenu, les étudiants deviennent, à leur tour, destinataires intempestifs de cette lettre. La postérité, pour le coup, ne s'y est pas trompée ; cette circulaire méritait bien le nom de «lettre », désignation de ce qui s'y agitait vraiment : une parole qui, en dépit de ses défauts, mais peut-être aussi en vertu de ceux-ci, délivre, comme dans une bouteille jetée à la mer, un message qui demande encore à être lu et interprété - quand, inversement, l'arrêté de mai $2010{ }^{11}$ portant définition des « dix compétences que doivent acquérir, etc. » saura bien se contenter de sa dénomination administrative précisément parce qu'il ne s'adresse à personne.

\section{IV. Épilogue : 2004-2011 - et après ?}

C'est ce que l'on appelle couramment un comble : un texte qui, sans dédaigner l'emphase, proclame dans son intitulé l'injonction faite au fonctionnaire d'État de se conduire en sujet éthique et pour qui les sujets ou, pour le dire en termes plus sociologiques, les acteurs n'existent pas - sinon comme des êtres de papier. Car il n'y a ici que «l'enseignant générique " - qui «connaît " (en vrac et de manière non exhaustive : les valeurs de la République, les mécanismes économiques, la politique éducative de la France...) ; qui est " capable » (de se situer dans la hiérarchie de l'institution scolaire, de participer à la vie de l'école ou de l'établissement, etc.); ou encore que son souci d'" agir de manière éthique et responsable conduit à », par exemple, « respecter les élèves et leurs parents ». Louable rappel, dont la crédibilité se heurte cependant au fait que, dans le même temps, ces «élèves » et ces "parents» ne sont tolérés, eux aussi, que comme de pures abstractions. Le "père de famille » dont Jules FERRY pensait, non sans naïveté (ou calcul), que, dans sa classe, l'instituteur était le "suppléant», faisait probablement davantage l'objet de considération: se soucier de ne pas choquer ses convictions enveloppait nécessairement l'idée de sa singularité (quand bien même on ne doutait pas que celle-ci fût soluble sans reste dans une morale opportunément « universelle »). Et sans doute estce par là que le discours de Jules FERRY reste tourné vers nous : c'est précisément parce que l'univocité (supposée, voire rêvée) du "père de famille » a laissé place à l'irréductible diversité des familles que l'invitation au tact formulée en 1883 gagne aujourd'hui une actualité d'autant plus forte qu'elle est celle de la complexité d'une société pluriculturelle - mais aussi d'une société concurrentielle, au sein de laquelle l'école a acquis un poids extraordinaire dans le destin des individus. Tous les parents le savent, sans doute, mais tous ne savent pas comment dire aux enseignants les espoirs qu'ils placent dans l'école même, et peut-être surtout, lorsque leurs enfants, pour des raisons multiples, revendiquent sur un mode «ethnicisé » ou " racialisé » l'expérience négative qu'ils font de l'univers scolaire et, au-delà, du monde social en général (c'est-à-dire, aussi, d'euxmêmes), jusqu'à produire, à l'avenant, des anathèmes, des stigmatisations, voire des violences inadmissibles au regard du projet d'institution d'un monde commun ${ }^{12}$. Quoi qu'il en soit, ce que savent les enseignants, et ce qu'attestent les inventions, précaires mais essentielles, dont ils font montre au quotidien notamment dans le domaine de la relation famille / école, c'est que rien n'est possible si, quel que soit le « respect » déclaré, on considère l'autre comme l'échantillon représentatif d'une "culture» ou d'une « communauté » supposées uniformes, au lieu de s'adresser à un individu. Autrement dit : 
au lieu de défendre le droit, pour chacun, de ne pas se laisser définir par ses origines, le droit de ne pas coïncider avec la somme des déterminations (familiales, culturelles, sociales) dans lesquelles il est d'abord nécessairement pris. N'est-ce pas là précisément la signification majeure de la liberté de conscience - et par là du principe même de laïcité ?

C'est même là, au vrai, que réside un des arguments les plus forts de ceux qui, dès les années 1990, soulignant que les mineurs ne sont pas encore en pleine possession des aptitudes intellectuelles et psychologiques nécessaires à l'exercice du libre examen ${ }^{13}$, ont été partisans d'une interdiction stricte du port de signes religieux à l'école ${ }^{14}$. Certes, après que, en 2004, une loi a statué en ce sens, faisant ainsi porter l'interdiction non plus seulement sur le port ostentatoire de signes religieux (comme le préconisait la circulaire BAYROU du 20 septembre 1994) mais sur le port de signes religieux ostensibles, la contradiction guettant cette argumentation apparaît nécessairement : c'est que, comme l'écrit Jean-Claude MONOD, « est prônée, en dernière instance, l'exclusion, hors de ce lieu d'émancipation [l'école publique], des mineurs qu'il s'agirait d'émanciper » (MONOD, 2007, p. 131). La contradiction touche bien à la " chose même » : l'attachement, constitutif de la laïcité, à l'autonomie du sujet. Que les conditions intellectuelles, psychologiques, voire morales de celle-ci ne soient encore qu'ébauchées chez les individus mineurs, on l'admettra aisément. Mais cela même suppose qu'il existe déjà quelque chose comme un sujet capable de recevoir les contenus qui précisément doivent lui permettre de former de telles aptitudes - et par là de transformer le regard qu'il porte sur les conduites, pratiques et convictions dont il a d'abord hérité. Or c'est bien, en fin de compte, la possibilité d'une telle dynamique, certes nullement garantie (ni quant à son déclenchement, ni quant à son issue : décrète-t-on l'« émancipation » d'autrui ${ }^{15}$ ?) qu'efface le glissement du curseur, en matière d'interdiction, de l'ostentatoire à l'ostensible. Jean ВАUвÉROT, membre de la commission STASI, a bien relevé ce point lorsqu'il s'est expliqué sur son refus de voter la proposition qui devait devenir le contenu essentiel de la loi du 15 mars 2004 : interdire, sous peine d'exclusion, le port de signes religieux ostensibles à l'école, c'est en réalité fixer a priori leur sens, et du même coup nier la pluralité de significations dont les "porteurs » sont susceptibles de les investir. "Ostensible», un signe religieux ne sera donc jamais, à la lettre, inoffensif, mais signifiera toujours l'intention d'offenser - mais si la volonté de montrer un signe en vue de provoquer, voire de nuire à autrui est ainsi littéralement pré-jugée, quelle consistance une distinction comme celle de l'ostensible et de l'ostentatoire peut-elle encore détenir ? Qu'est-ce donc que cette volonté de personne ? À cet égard, le fait que la loi encadrant le port des signes religieux à l'école ait été si rapidement connue sous le nom de «loi sur le voile » délivre sa troublante vérité : les jeunes filles musulmanes, qu'on dit tant vouloir émanciper, ne sauraient être des sujets (et dans ce présupposé se répète la logique même de la domination, en particulier de la domination masculine, qu'on prétend combattre). Elles sont, semble-t-il, destinées à être moins au fait des « intentions » qu'elles attachent (ou non) à leur manière de se vêtir que, manifestement (ostensiblement?), le sont ceux qui savent fixer une fois pour toutes la signification de leur tenue. En fin de compte, les voilà définies par cette rareté : un signe clos sur lui-même, dont le sens ne s'établit guère par le rapport avec d'autres signes c'est-à-dire des attitudes, des comportements, bref, des interactions infiniment diverses dans le jeu desquelles il nous semble quand même un peu téméraire (ou malhonnête) de ne pas lire aussi des « signes » d'émancipation ${ }^{16}$.

Aussi, et non pas, certes, seulement : l'angélisme n'est pas de mise, et il n'est nullement question d'admettre que, portant ou non, d'ailleurs, des «signes religieux ostensibles", 
des élèves, au nom de leurs convictions (et, soit dit en passant, pourquoi ne pas avoir également légiféré sur le port ostensible de signes non religieux?), enfreignent les règlements intérieurs des écoles ou des établissements, violentent moralement ou physiquement des camarades, voire des personnels, ou encore refusent de suivre certains enseignements. Notre propos est autrement prudent: il ne vise qu'à établir les coordonnées conceptuelles d'un problème, tel qu'il commande, par excellence, le sens et la fonction que la loi de 2004 accorde à une « mise en œuvre [qui] passe d'abord par le dialogue » (circulaire du 18 mai 2004 relative à la mise en œuvre de la loi no 2004-228 du 15 mars 2004, section III). Ce problème, on peut tenter de le formuler en ces termes : si le texte précise que l'exclusion ne doit avoir lieu qu'en dernier recours, et par conséquent préconise le dialogue avec l'élève, il verrouille nécessairement l'objet de ce dialogue même. Car l'échange ne se déroulera jamais que sur le fond d'un présupposé indiscutable : la signification du signe religieux " ostensible » est le prosélytisme ; on cherchera donc à convaincre (avec "pédagogie », en sollicitant la médiation de l'équipe pédagogique ou éducative, etc.) l'élève de retirer le signe incriminé - mais jamais à discuter du sens que, dans l'espace de ses interactions avec les élèves et adultes qui l'entourent, il ou elle lui confère.

Il est cependant remarquable - et c'est pourquoi il s'agit rigoureusement, ici, d'un problème - que le texte de cette même loi de 2004 signale par endroits des réserves quant à sa propre logique (comme en témoignent aussi, du reste, les préconisations du rapport STASI qui n'ont finalement pas été retenues). Si le terme "ostensible " possède encore un minimum de sens, alors il s'oppose à « discret ». Mais l'opposition entre signes ostensibles et signes discrets à laquelle il faut alors faire droit (de manière plus ou moins explicite) trahit précisément le fait qu'il était impossible de dissimuler tout à fait le coup de force sémantique par lequel on a identifié l'ostensible à l'ostentatoire. Car l'ostentation n'a en fin de compte de sens qu'à traduire la manière dont un individu choisit non pas seulement une tenue, mais se choisit lui-même dans le réseau de ses conduites - lesquelles sont en effet susceptibles de conférer à son apparence vestimentaire telle ou telle signification. Comment, dès lors, ne pas accorder que, même discret, un signe religieux (et, encore une fois, pourquoi seulement « religieux » ?), peut se révéler, selon la manière dont l'individu s'y rapporte, dérangeant? Quand vient le moment de décrire précisément le «Champ d'application de la loi », c'est bien ce que la rédaction, quelque peu contournée, enregistre de façon symptomale :

La loi ne remet pas en cause le droit des élèves de porter des signes religieux discrets. [...] En revanche, la loi interdit à un élève de se prévaloir du caractère religieux qu'il y attacherait, par exemple, pour refuser de se conformer aux règles applicables à la tenue des élèves dans l'établissement (circulaire du 18 mai 2004, section II, \& 2.1).

De manière analogue, le texte admettait que les intervenants occasionnels (dont, bien entendu, les parents) ne soient pas soumis à l'interdiction du port de tout signe religieux, même discret, qui s'impose aux agents du service public d'éducation. Si "problème » il y a, donc, dans ce texte, si la rédaction de celui-ci fait parfois "symptôme", cela reste précisément à mettre à son crédit : la persistance de l'ambiguïté est la trace de l'attention que, des débats menés au sein de la commission STASI jusqu'à l'élaboration de la loi, on prêta malgré tout à la complexité des choses ${ }^{17}$. Considérée dans cette perspective, la législation de 2004 fournit un repère à partir duquel on peut faire apparaître le sens des évolutions qui ont eu lieu jusqu'aujourd'hui. Dire que ce sens est celui d'un abaissement intellectuel n'est pas trop fort. 
30 Expliquons-nous. Pour autant que l'on puisse s'approprier de manière féconde un intitulé comme "Agir en fonctionnaire de l'État et de manière éthique et responsable ", c'est dans la mesure où l'on identifiera, dans la répétition de la conjonction «et », la possibilité insistante de la disjonction: celle, précisément, qui peut toujours survenir entre l'adhésion sincère des acteurs (ici: les personnels d'éducation) aux valeurs et aux principes républicains qui fondent leur engagement professionnel et la reconnaissance du fait que, au sein des situations concrètes les plus diverses, ces références-là ne suffisent pas à déterminer la règle de leur pratique. Là commence, si l'on veut, l'« éthique »: lorsque, au sein d'interactions complexes, c'est l'attachement même à nos exigences morales qui nous fait éprouver les limites de notre maitrise, et que l'essentiel tient alors à des choix toujours risqués, mettant notre "sagacité herméneutique» (selon un mot d'Eirick PRAIRAT) au service d'une action dont nous voulons en effet, tant au plan de ses principes qu'au regard de ses conséquences, pouvoir répondre sans jamais en être pleinement assurés (le sentiment d'être « responsable » n'indique pas autre chose).

31 On comprend alors que, confrontés à la difficulté de la tâche, étudiants et enseignants puissent parfois rester, disons, interdits devant la rédaction de la "compétence 1 ", mélange inédit (dans sa forme du moins) de rhétorique administrative et de bonnes intentions - quelque chose comme une variante bureaucratique de ce que Jacques ROUBAUD nomma un jour la «langue Muesli». En l'occurrence, le scepticisme constitue encore le meilleur hommage à rendre aux valeurs que, célébrant les noces du républicanisme bon teint et de la platitude techniciste, un tel jargon défigure. Il y va à la fois d'un réflexe de protection et d'une forme de résistance opposés à l'agression que représentent, désormais, des injonctions si troubles et contradictoires que ceux qui les reçoivent se demandent comment, et combien de temps, ils pourront les supporter sans rompre. Entendons : sans rester béants, étourdis par la honte de se sentir inexorablement enrôlés au service d'une option politique déterminée. "Respecter les familles ", (nous) assène-t-on. Certes. Mais qu'est-ce que cela signifie lorsque le parti présidentiel, suivi en cela, on l'a vu, par le ministre de l'Éducation nationale, propose, en vue de « réaffirmer le principe de laïcité dans le cadre des services publics ", d'interdire désormais aux femmes portant un foulard d'accompagner la classe de manière occasionnelle ou régulière, ou encore de participer à divers conseils et réunions d'école ou d'établissement? Pourquoi, il est vrai, attendre d'elles qu'elles puissent ne pas être une menace pour la laïcité puisque, se plaçant explicitement dans la lignée du rapport BAROIN de 2003, celui de 2011 a retenu, n'est-ce pas, qu'il n'y a pas d'individus musulmans, seulement des échantillons de « certaines populations immigrées " qui, " en particulier dans le monde musulman », sont "issues d'une culture non laïque et non démocratique » et ne sauraient, dès lors, "[percevoir] le sens $[\mathrm{du}]$ principe de laïcité ». Assurément, les parlementaires UMP qui intitulent très sérieusement leur rapport Laïité. Pour mieux vivre ensemble (et, pour faire bonne mesure, le placent sous l'égide du " pacte républicain ») s'emploient sans trêve ni repos à réaliser cette vision fort inspirée d'une "laïcité culturelle et identitaire " qui, étayée par des considérations socio-anthropologiques de haut vol sur la « culture musulmane », faisait le sel inimitable (du moins osait-on l'espérer) de la prose de M. BARoIN. In fine, le tout donne la recette de l'abêtissement en politique : d'un côté, là où il faudrait penser à partir d'eux, de leurs expériences, de leurs inventions, de leurs incertitudes aussi, les individus sont effacés (derrière la référence incantatoire aux «cultures » et aux "communautés ») ou déniés (les personnels d'éducation, comme au reste bien des acteurs des milieux de la santé, de la culture, du travail social, entendent d'autant plus résonner les appels à la 
« responsabilité » que le cadre normatif censé guider leur action se disloque, les laissant assujettis à des accès d'anxiété, sinon de mépris de soi, confinant parfois à la pathologie). De l'autre, quelque individu dont les hautes charges impliquent pourtant un souci exemplaire de l'intérêt général se complaît dans l'hypertrophie de la subjectivité. Un président de la République qui, en octroyant un statut d'exception à la « chrétienté » dans la détermination de "l'identité de la France », affirme le principe de ce fractionnement communautaire de la société qu'il affecte cependant de dénoncer, celui-là ne se contente pas de déduire la physionomie de la sphère publique de ses préférences confessionnelles : ce faisant, il va jusqu'à récuser l'attachement à la préséance du bien de l'état sur le sien propre - cette vertu dont MONTESQUiEU a dit combien elle est indispensable au gouvernement républicain (MONTESQUIEU, 1979 [1748], p. 143 sq.).

Aussi devient-il impossible, aux yeux du «fonctionnaire de l'État» qui, sachant l'importance d'un tel principe, en reconnaît une traduction précieuse dans la neutralité laïque, d'esquiver ce constat: "Agir de manière éthique et responsable » constitue la formule d'une ambition bien intrépide à une époque où, dans les allées du pouvoir et jusque sous les ors de la République, on ne recule guère devant les conduites partiales et irresponsables.

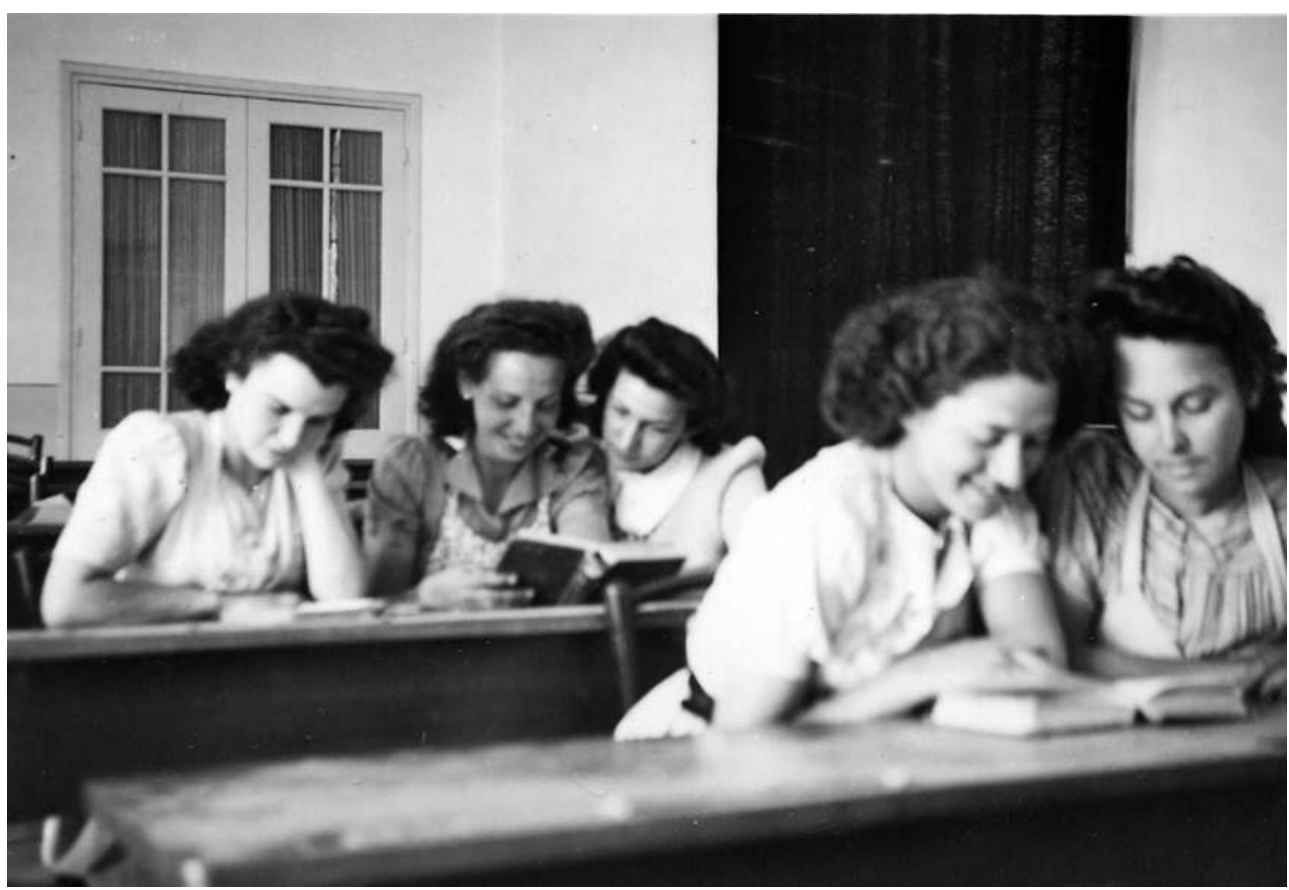

Elèves institutrices dans la salle d'étude de l'Ecole normale Eckmühl, Oran, 1940 (archives familiales de Gilles MOUTOT).

Peu de temps après que cette photographie a été prise, les deux jeunes filles assises au premier plan furent victimes de la politique d'exclusion menée par le régime de Vichy à l'encontre des Juifs. Grâce au soutien de la directrice de l'Ecole, Mme Tubiana, et aux contributions matérielles de quelques familles de pensionnaires, elles purent néanmoins poursuivre leur scolarité pendant quelques mois avant que l'Etat français ne procède, à partir du $1^{\text {er }}$ octobre 1941, à la fermeture des Ecoles normales. 


\section{BIBLIOGRAPHIE}

ADORNo (Theodor W.), « Sujet et objet » (1970, posth.), Modèles critiques, trad. fr. É. KAUfHOLZ et M. JIMENEZ, Paris, PAYOT, 1984.

BAUBÉROT (Jean), « Laïcité, le grand écart », Le Monde du 3 janvier 2004.

BAUвÉROT (Jean), « La gauche doit refuser la laïcité identitaire de SARKOZY », Libération du 29 mars 2011.

CONDORCET (Jean-Antoine-Nicolas), Rapport et projet de décret sur l'organisation générale de l'instruction publique (1792), Euvres de Condorcet, Paris, FIRMIN-DIDOT, 1847, édition numérisée disponible en ligne sur Google Books :

http://books.google.fr/books?id=Nc0TAAAAQAAJ\&vq=ce+n\%27est+pas+un+véritable +droit\&hl=fr\&pg=PP9\&redir_esc=y\#v=onepage\&q=ce\%20n'est\%20pas\%20un\%20véritable\% 20droit\&f=false

DEBRAY (Régis), Le Moment fraternité, Paris, GALLIMARD, 2009.

DEBRAY (Régis), Éloge des frontières, Paris, GALLIMARD, 2010.

FASSIN (Éric), « Les deux sexes de la droite », Libération du 7 septembre 2011.

FERRY (Jules), « Lettre aux instituteurs » (1883), in IGEN (dir.), L’Idée républicaine aujourd'hui. Guide républicain, Paris, DELAGRAVE, 2004, pp. 235-241.

KINTZLER (Catherine), La République en questions, Paris, MINERVE, 1996.

KINTZLER (Catherine), Qu'est-ce que la laïcité ?, Paris, VRIN, 2007.

MONOD (Jean-Claude), Sécularisation et laïcité, PUF, coll. « Philosophies », 2007.

MONOD (Jean-Claude), « L’abandon de la neutralité laïque », Le Monde du 29 janvier 2008.

MONTESQUIEU (Charles-Louis de SECONDAT), De l'esprit des lois (1748), Paris, GF, 1979.

PENA-RUIZ (Henri), La Laïcité, Paris, FLAMMARION, 2001.

RANCIÈRE (Jacques), La Haine de la démocratie, Paris, LA FABRIQUE, 2005.

\section{NOTES}

1. Et non la Haute Autorité de lutte contre les discriminations et pour l'égalité (HALDE) - note du rédacteur.

2. Ou encore du programme de terminale L qui inscrit, à partir de la rentrée 2012, l'étude des familles homoparentales, au même titre que les familles monoparentales et recomposées, au programme de la spécialité « Droits et enjeux du monde contemporain ».

3. «Monsieur le ministre, nous ne pouvons accepter que l'école devienne un lieu de propagande, où l'adolescent serait l'otage de préoccupations de groupes minoritaires en mal d'imposer une vision de la "normalité" que le peuple français ne partage pas » (lettre ouverte du 31 mai 2011 adressée par Mme Christine Boutin au ministre de l'Éducation nationale). 
4. Première des dix compétences à acquérir par les professeurs, documentalistes, conseillers principaux d'éducation, pour l'exercice de leur métier (arrêté du 12 mai 2010).

5. Ibid.

6. Il y aurait certes à critiquer de façon détaillée l'emploi de ce terme, si lâche et irréfléchi qu'il fait perdre au concept de compétence toute la rigueur et la fécondité qu'ont pu lui conférer, en philosophie et en sociologie, les traditions empiristes ou pragmatistes.

7. Parmi les nombreuses éditions de ce texte, on peut se reporter à la reproduction qui figure dans l'ouvrage publié sous la direction de l'Inspection générale de l'Éducation nationale : L'Idée républicaine aujourd'hui. Guide républicain, DELAGRAVE, 2004, pp. 235-241.

8. Lors de la dernière rentrée scolaire, le ministre de l'Éducation nationale s'en serait voulu de rater le coche. Aussi s'est-il empressé de reprendre l'antienne déjà immortalisée par son prédécesseur immédiat en annonçant, dès la fin du mois d'août, le «retour de la morale à l'école» (mais quand donc s'en était-elle absentée ?).

9. Voir, à ce propos, les précisions données ici même par Yves VERnEUIL dans son article : «L'école et la laïcité, de l'Ancien Régime à nos jours : enjeux du passé, enjeux dépassés ?»

10. Voir sur ce point, dans le présent volume, l'entretien avec François DUBET.

11. Ou encore l'annexe du BOEN nº 29 du 22 juillet 2010.

12. Voir également, dans l'entretien cité à la note 10, les mises au point de François DUBET sur ces questions.

13. Qu'on pense par exemple à des textes importants de Catherine KINTZLER, comme La République en questions, MINERVE, 1996, ou Qu'est-ce que la laïcité ?, VRIN, 2007.

14. En opposition, d'ailleurs, aux avis rendus par la cour de cassation. Le cycle jurisprudentiel des années 90, accompagnant les diverses affaires dites « du voile» (depuis le cas de Creil, en 1989), tranche en effet à chaque fois en faveur des voilées et de leurs familles (et donne lieu en 1994 à la circulaire BAYROU).

15. À vrai dire, la réponse à une telle question engage une prise de position quant aux ambivalences du projet républicain lui-même. Traiter convenablement de ce point exigerait un travail d'une tout autre ampleur que le présent article.

16. Comme l'a bien rappelé Jean BAUBÉROT : «Une scolarité plus longue, un âge de mariage plus tardif, moins d'enfants que la génération précédente, l'entrée dans le marché du travail : tous les indicateurs d'émancipation sont réunis pour les jeunes femmes de conviction ou de culture musulmane. C'est pour cette raison qu'elles font trop souvent face à un machisme exacerbé. Les aider à passer ce cap difficile nécessite de savoir que toutes ne s'émancipent pas de la même manière et que certaines peuvent le faire en portant, sans agressivité, un signe "ostensible", adjectif qui signifie - selon Le Petit Robert: "Qui peut être montré publiquement sans inconvénient"! » (BAUBÉROT, 2004).

17. C'est là un point que Jean-Claude MONOD a très précisément cerné : «La décision d'interdire les "signes religieux manifestant ostensiblement une appartenance religieuse" dans les écoles ne se déduit [pas] nécessairement des principes fondateurs de la laïcité. Ce qui ne veut pas dire qu'on ne puisse justifier cette décision par d'autres raisons, momentanées et contingentes, tenant à des rapports de force ou à des motifs d'ordre public - mais il importe de distinguer le noyau essentiel des principes de la marge d'ajustement pragmatique à des contextes théologicopolitiques en évolution » (MONOD, 2007, p. 131-132). 


\section{RÉSUMÉS}

Le Code l'éducation indique que, dans l'exercice de leurs missions, les personnels mettent en œuvre les valeurs de la République. La laïcité est l'une d'elle, inséparable de la trilogie républicaine. Mais comment la mettre en œuvre, quand s'exposent des conceptions de la laïcité aussi difficilement compatibles que celle proposée dans le rapport de la commission de réflexion sur l'application du principe de laïcité dans la république (STASI, décembre 2003) et celle développée par le président de la République au Puy-en-Velay le 3 mars 2011 ?

Confrontant en outre les évolutions récentes avec quelques références majeures dans l'histoire des rapports entre école républicaine et laïcité (ainsi de CONDORCET ou de FERRY), on se demande ici comment le professeur ou conseiller principal d'éducation, appelé à « agir en fonctionnaire de l'État et de manière éthique et responsable ", pourrait aujourd'hui exercer cette compétence autrement qu'en se référant à une éthique personnelle et à un bricolage conceptuel. Or une telle éthique est-elle, malgré sa nécessité, envisageable dans ce contexte?

The French Code of Education points out that, while they accomplish their professional tasks, public servants bring values of the Republic into play. Indissolubly linked to the Republican trilogy, secularism ("laïcité") is one of those. But how to bring it into play when some ideas of secularism that can very hardly match themselves are put forward - for instance the idea which is implied by the report concerning the way to apply the "secularism principle" (the STASI Committee Report, December 2003) and the one that the President of the French Republic developed in his speech of the March 3rd 2011, in Le Puy-en-Velay?

Furthermore, by comparing these conceptions to milestones in the history of the relations between Republican school and the secularism (like CONDORCET's or FERRY's discourses), we ask ourselves in this paper how teachers, school librarians, pastoral leaders, school managers, who are summoned to "behave as a State civil servant and in an ethical and responsible way", could exercise their ability in that matter by doing anything different than turning themselves to personal ethic and a conceptual "do-it-yourself". But, as necessary as it might be, is such an ethic thinkable in that context?

\section{INDEX}

Mots-clés : compétence, Condorcet, école, éthique, Ferry, laïcité, république

Keywords : competence, Condorcet, ethics, Ferry, republic, school, secularism

\section{AUTEURS}

\section{GILLES MOUTOT}

Formateur à l'IUFM de Montpellier - Université Montpellier 2 


\section{JEAN-PIERRE VÉRAN}

Formateur associé à l'IUFM de Montpellier - Université Montpellier 2 